\title{
Effects of Insulin on Gestational Pima Indian Heritage Females
}

\author{
Ishita Saha ${ }^{1}$, Debjit Konai ${ }^{2}$, Sunit Kumar Medda ${ }^{3}$ and Rabindra Nath Das ${ }^{2 *}$ \\ ${ }^{1}$ Department of Physiology, Medical College and Hospital, Kolkata, W.B., India. \\ ${ }^{2}$ Department of Statistics, the University of Burdwan, Burdwan, West Bengal, India. \\ ${ }^{3}$ Kalyani J.N.M. Hospital, Kalyani, Nadia, West Bengal, India.
}

*Corresponding Author: Rabindra Nath Das, Department of Statistics, the University of Burdwan, Burdwan, West Bengal, India.

\section{Received Date: 16 September 2021 | Accepted Date: 08 October 2021 | Published Date: 16 October 2021}

Citation: I Saha, D Konai, Sunit K Medda, Rabindra N Das. (2021) Effects of Insulin on Gestational Pima Indian Heritage Females. Endocrinology and Disorders. 5(7): DOI:10.31579/2640-1045/100

Copyright: (C) 2021 Rabindra Nath Das, This is an open-access article distributed under the terms of the Creative Commons Attribution License, which permits unrestricted use, distribution, and reproduction in any medium, provided the original author and source are credited.

\begin{abstract}
:
Diabetes and obesity reach epidemic proportions all over the world, while the effects of insulin resistance and its consequences are gaining prominence. The article derives the effects of insulin on gestational Pima Indian heritage females with minimum 21 years old. It is identified herein that mean insulin level for gestational women $(\mathrm{GW})$ is positively linked with glucose level $(\mathrm{P}=0.0055)$, while it is negatively linked with age $(\mathrm{P}=0.0095)$. Mean insulin level for $\mathrm{GW}$ is independent of pregnancies $(\mathrm{P}=0.1866)$ and diabetes pedigree function (DPF) $(\mathrm{P}=0.5321)$, while it is partially positively linked with their joint interaction effect Pregnancies* DPF $(\mathrm{P}=0.0864)$. It is positively linked with blood pressure $(\mathrm{BP})(\mathrm{P}<0.0001)$ and triceps skin-fold thickness (TST) $(\mathrm{P}<0.0001)$, while it is negatively linked with the joint interaction effects $\mathrm{BP} * \mathrm{TST}(\mathrm{P}<0.0001)$ and DPF*TST $(\mathrm{P}<0.0001)$. In addition, mean insulin level is negatively linked with body mass index $(\mathrm{BMI})(\mathrm{P}=0.0001)$, while it is positively partially linked with the interaction effect BMI*DPF $(\mathrm{P}=0.1312)$. Variance of insulin level is positively linked with pregnancies $(\mathrm{P}=0.0184)$ and age $(\mathrm{P}=0.0027)$, while it is negatively linked with their joint interaction effect Pregnancies*Age $(\mathrm{P}=0.0418)$. Also variance of insulin level is partially negatively linked with $\mathrm{BMI}(\mathrm{P}=0.0738)$ and glucose level $(\mathrm{P}=0.1439)$, while it is partially positively linked with their joint interaction effect $\mathrm{BMI}{ }^{*} \mathrm{Glucose}(\mathrm{P}=0.1472)$. Further, variance of insulin level is negatively linked with $\mathrm{DPF}(\mathrm{P}=0.0309)$. It is concluded that for $\mathrm{GW}$, insulin level increases with the increase of glucose level, BP, TST, and the interaction effects Pregnancies*DPF \& BMI*DPF, and it decreases with the increase age, BMI, and the interaction effects BP*TST \& DPF*TST. GW should be careful on her glucose level, BMI, BP \& TST
\end{abstract}

Key Words: body mass index (BMI); gestational women $(\mathrm{GW})$; glucose level; insulin level; joint generalized linear gamma models; triceps skin fold thickness (TST)

\section{Introduction}

The worldwide escalation of diabetes and obesity in developing and developed countries poses a great health challenge. BMI, or obesity is one of the principal causes of type 2 diabetes. Type 1 diabetes is mainly due to the autoimmune-mediated waste of pancreatic beta cells leading to insulin deficiency [1-3]. The lack of sufficient insulin secretion reveals all hyperglycemic states. If the insulin action is not normal for type 1 diabetes, there is a near total insulin secretory loss function. The insulin secretion abnormalities are multiple for type 2 diabetes. One of the primary defects is an early phase loss of meal-stimulated insulin secretion. This is due to the inability of the beta-cell to increase sufficient insulin secretion to overcome peripheral and hepatic insulin resistance [4-6]. Type 2 diabetes is discriminated against by a progressive loss in both secretory function and beta-cell mass and so that for most persons, absolute insulin deficiency appears in the late disease stages.
Commonly, type 1 , gestational, type 2 diabetes are observed in practice in our society. Due to some unusualness of human body components, if the pancreas is unable to produce insulin, type 1 diabetes occurs that is known as juvenile diabetes [4, 5-7]. During pregnancy time, it is commonly observed that glucose levels become higher for some $\mathrm{GW}$, and they are affected with diabetes, which is known as gestational diabetes (GD). Afterwards, the GD is reduced to type 2 diabetes [3, 4, 8-10]. Type 2 diabetes patients commonly feel the following complications. One complication is that human body components can't produce sufficient insulin, and the second complication is that the sufficient produced insulin can't do its regular functions properly, which is called insulin resistance [10-12]. The human organ produces sufficient insulin, while the insulin impressibility is unidentified, and it does not function as it should do, and the glucose is not entering the body's cells sufficiently. Consequently, blood sugar level rises in the human body, and the cells are not accepting their required nutrients for necessary growth and energy [11-14]. 
The explanatory factors of insulin level are little focused in the previous articles $[7,9,10]$. The insulin level's explanatory factors will be different for type 1, GD, type 2 diabetes patients. Based on the knowledge of insulin level's explanatory factors, medical practitioners can take necessary actions on the diabetes patients. This article focuses on the effects of insulin on some gestational Pima Indian heritage females. The following hypotheses related to GW are examined in the current article based on a real data set. The aimed hypotheses are: (1) are there any effects of insulin level on gestational Pima Indian heritage females?, (2) if they are affirmative, what are the effects of insulin level on the GW? The article is ordered as follows. The subsequent section presents materials \& methods, and the rest sections are statistical \& graphical analysis, outcomes \& discussion, and conclusions.

\section{Materials \& Methods}

\section{Materials}

The article is prepared with the help of a real data set associated with Pima Indian heritage of 768 gestational females at least 21 years old. The aimed dataset was initially collected by the National Institute of Diabetes and Digestive and Kidney Diseases (NIDDKD). The data set can be found in the UCI Machine Learning Repository. It counts 9 explanatory study variables such as body mass index (BMI), age (in years), plasma glucose concentration level over 2 hours in an oral glucose tolerance test (Glucose), diastolic blood pressure (BP) $(\mathrm{mm} \mathrm{Hg})$, investigation unit type (IUT) (1=non- diabetic, $2=$ diabetic), number of pregnancies (NOP), triceps skin-fold thickness (TST) (mm), diabetes pedigree function (DPF), 2-hours serum insulin (mu U/ml) (Insulin), Here all the explanatory variables are continuous except IUT, which is an attribute character. It is noted that DPF is a function that estimates diabetes likelihood depending on family history.

\section{Statistical Methods}

The present gestational females data set is physiological data that is of a commonly heteroscedastic nature. The response insulin level is heteroscedastic positive and continuous. Unequal variance response insulin level can be modeled by adopting a suitable transformation when the insulin level variance is stabilized by the transformation. Commonly, the response variance may not be stabilized in several cases [15]. It is known that a positive continuous constant variance response variable can be modeled either by the gamma, or the lognormal model [16]. For a nonconstant variance continuous positive response variable, modeling can be suitably done using joint generalized linear models (JGLM) under the lognormal, or the gamma models [17-20]. JGLMs is illustrated in the book by Lee et al. [18]. The response insulin level is modeled in the article using the joint gamma models, so it is shortly reported herein.

\section{JGL gamma models}

The dependent variable herein insulin level is modeled with the remaining variables. Let us consider Insulin level $=\mathrm{y}_{\mathrm{i}}$ as the random response variable with mean $\mu_{\mathrm{i}}=\mathrm{E}\left(\mathrm{y}_{\mathrm{i}}\right)$ and non-constant variance $\left(\sigma_{i}{ }^{2}\right)$, maintain $\operatorname{Var}\left(\mathrm{y}_{\mathrm{i}}\right)$ $=\sigma_{i}^{2} \mu_{\mathrm{i}}^{2}=\sigma_{i}^{2} V\left(\mu_{i}\right)$ say, where $V($.$) is termed as the variance$ function that recognizes generalized linear models (GLMs) family distribution. For instance, if $V(\mu)=\mu$, it is Poisson, and it is Normal, or gamma as $V(\mu)=1$, or $V(\mu)=\mu^{2}$ etc.

Mean $\&$ dispersion JGLMs for insulin level with gamma distribution are displayed as

$\eta_{i}=g\left(\mu_{i}\right)=x_{i}^{t} \beta$ and $\varepsilon_{i}=h\left(\sigma_{i}^{2}\right)=w_{i}^{t} \gamma$,

where $g(\cdot) \& h(\cdot)$ are the GLM link functions for the mean \& dispersion linear predictors respectively, and $x_{i}{ }^{t}, w_{i}{ }^{t}$ are the independent study variables vectors associated with the mean and dispersion parameters respectively. Maximum likelihood (ML) method is considered to estimate mean parameters, while the restricted ML (REML) method is applied to estimate dispersion parameters [18].

\section{Statistical \& Graphical Analysis}

The dependent variable insulin level is modeled on the remaining independent variables adopting JGLMs under gamma distribution, as the gamma fit gives better results than the Log-normal fit. Independent variables are age, glucose, TST, IUT, DPF, BP, NOP, BMI. The independent variable insulin level is marked as heteroscedastic, so it is modeled by applying JGLMs under gamma distribution. The best selected insulin level joint gamma fitted model is accepted based on the lowest Akaike information criterion (AIC $=7195.529)$ value, which lowers both the predicted additive errors and squared error loss [21; p. 203-204]. The best insulin level joint gamma fitted analysis findings are displayed in Table 1. Lower order effects such as pregnancies, DPF (in the mean model) and glucose (in the dispersion model) are included in the model even if they are insignificant, as their higher order interaction effects are significant due to the marginality rule by Nelder [22]. For better fitting, some partially significant effects such as BMI*DPF, Glucose*BMI are included in the model, which are called confounders in Epidemiology [21].

\begin{tabular}{|c|c|c|c|c|c|}
\hline Model & Covariate & estimate & $\begin{array}{l}\text { Standard } \\
\text { error. }\end{array}$ & $\mathbf{t}(\mathbf{7 5 6})$ & P-value \\
\hline \multirow[t]{12}{*}{ Mean } & constant & -1.9302 & 1.4414 & -1.339 & 0.1810 \\
\hline & Glucose (x2) & 0.0162 & 0.0058 & 2.782 & 0.0055 \\
\hline & Pregnancies (x1) & -0.1342 & 0.1016 & -1.322 & 0.1866 \\
\hline & $\begin{array}{l}\text { Diabetes pedigree } \\
\text { Function }(\mathrm{DPF})(\mathrm{x} 7)\end{array}$ & 1.0677 & 1.7086 & 0.625 & 0.5321 \\
\hline & $\begin{array}{l}\text { Pregnancies } \\
(\mathrm{x} 1 * \mathrm{x} 7)\end{array}$ & 0.2541 & 0.1480 & 1.717 & 0.0864 \\
\hline & Age (x8) & -0.0518 & 0.0199 & -2.601 & 0.0095 \\
\hline & $\begin{array}{l}\text { Blood Pressure (BP) } \\
(\mathrm{x} 3)\end{array}$ & 0.0722 & 0.0112 & 6.453 & $<0.0001$ \\
\hline & $\begin{array}{l}\text { Triceps } \quad \text { Skin-fold } \\
\text { Thickness (TST) }(x 4)\end{array}$ & 0.3948 & 0.0445 & 8.864 & $<0.0001$ \\
\hline & $\mathrm{BP} * \mathrm{TST}(\mathrm{x} 3 * \mathrm{x} 4)$ & -0.0024 & 0.0006 & -4.157 & $<0.0001$ \\
\hline & DPF*TST $(x 7 * x 4)$ & -0.1417 & 0.0316 & -4.482 & $<0.0001$ \\
\hline & BMI (x6) & -0.1450 & 0.0371 & -3.906 & 0.0001 \\
\hline & DPF*BMI $(x 7 * x 6)$ & 0.0779 & 0.0515 & 1.511 & 0.1312 \\
\hline Dispersion & Constant & 3.5658 & 0.9590 & 3.718 & 0.0002 \\
\hline
\end{tabular}




\begin{tabular}{|l|l|l|l|l|l|}
\hline \multirow{7}{*}{} & Pregnancies (x1) & 0.1458 & 0.0617 & 2.363 & 0.0184 \\
\cline { 2 - 5 } & Age (x8) & 0.0244 & 0.0081 & 3.007 & 0.0027 \\
\cline { 2 - 5 } & $\begin{array}{l}\text { Pregnancies*Age } \\
(\mathrm{x} 1 * \mathrm{x} 8)\end{array}$ & -0.0032 & 0.0016 & -2.039 & 0.0418 \\
\cline { 2 - 5 } & BMI (x6) pedigree & -0.0524 & 0.0293 & -1.790 & 0.0738 \\
\cline { 2 - 6 } & $\begin{array}{l}\text { Diabetes } \\
\text { Function (DPF) (x7) }\end{array}$ & & 0.1557 & -2.162 & 0.0309 \\
\cline { 2 - 6 } & Glucose (x2) & -0.0119 & 0.0081 & -1.463 & 0.1439 \\
\cline { 2 - 5 } & Glucose*BMI (x6*x2) & 0.0003 & 0.0002 & 1.451 & 0.1472 \\
\hline AIC & $\mathbf{7 1 9 5 . 5 2 9}$ & & \\
\hline
\end{tabular}

Table 1: Joint gamma INSULIN level fitting mean and dispersion models

The insulin level gamma fitted JGLMs (Table 1) are diagnosed by Figure 1. Figure 1(a) displays the absolute insulin level gamma fitted residuals plot with respect to the predicted values, which shows that all points are randomly concentrated against a single point, except a point at the right

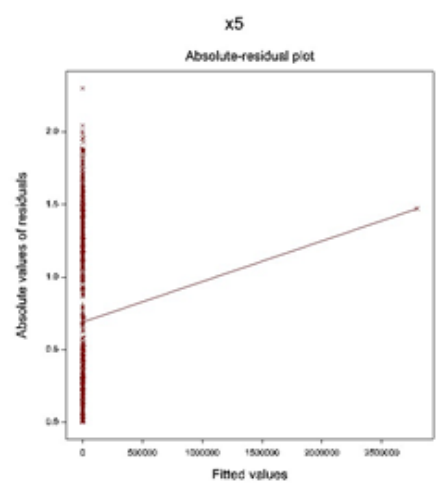

Figure 1(a) boundary. Figure 1(b) displays the insulin level gamma fitted mean model (Table 1) normal probability plot that does not display any lack of fit. The two plots Figure 1(a) and Figure 1(b) show that the insulin level joint gamma fitted models are appropriate.

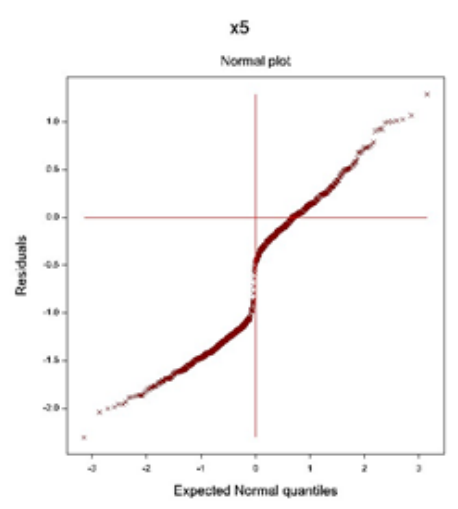

Figurel(b)

Figure 1: For the JGL gamma Insulin fit (Table 1), the (a) absolute residuals plot against the Insulin fitted values, and (b) the normal probability plot for the Insulin mean model.

\section{Results \& Discussions}

Insulin level JGL gamma fitted summarized results are presented in Table 1. It is identified herein that mean insulin level for GW is positively linked with glucose level $(\mathrm{P}=0.0055)$, while it is negatively linked with age $(\mathrm{P}=0.0095)$. Mean insulin level for $\mathrm{GW}$ is independent of pregnancies $(\mathrm{P}=0.1866)$ and DPF $(\mathrm{P}=0.5321)$, while it is partially positively linked with their joint interaction effect Pregnancies*DPF $(\mathrm{P}=0.0864)$. It is positively linked with BP $(\mathrm{P}<0.0001)$ and TST $(\mathrm{P}<0.0001)$, while it is negatively linked with the joint interaction effects $\mathrm{BP} * \mathrm{TST}(\mathrm{P}<0.0001)$ and DPF*TST $(\mathrm{P}<0.0001)$. In addition, mean insulin level is negatively linked with $\mathrm{BMI}(\mathrm{P}=0.0001)$, while it is positively partially linked with the interaction effect $\mathrm{BMI} * \mathrm{DPF}(\mathrm{P}=0.1312)$. Variance of insulin level is positively linked with pregnancies $(\mathrm{P}=0.0184)$ and age $(\mathrm{P}=0.0027)$, while it is negatively linked with their joint interaction effect Pregnancies*Age ( $\mathrm{P}=0.0418)$. Also variance of insulin level is partially negatively linked with $\mathrm{BMI}(\mathrm{P}=0.0738)$ and glucose level $(\mathrm{P}=0.1439)$, while it is partially positively linked with their joint interaction effect BMI*Glucose $(\mathrm{P}=0.1472)$. Further, variance of insulin level is negatively linked with $\mathrm{DPF}(\mathrm{P}=0.0309)$.

JGL gamma fitted insulin level mean $(\hat{\mu})$ model (Table 1$)$ is

$\hat{\mu}=\exp (-1.9302+0.0162$ Glucose --0.1342 Pregnancies $+1.0677 \mathrm{DPF}$ +0.2541 Pregnancies* DPF -- 0.0518 Age + 0.0722 BP + 0.3948 TST -$0.0024 \mathrm{BP} * \mathrm{TST}$-- 0.1417TST*DPF --0.145BMI + 0.0779 BMI*DPF),

and the JGL gamma fitted insulin level dispersion $\left(\hat{\sigma}^{2}\right)$ model (from Table $1)$ is $\hat{\sigma}^{2}=\exp (3.5658+0.1458$ Pregnancies +0.0244 Age -0.0032Pregnancies*Age -0.0524 BMI -0.3367 DPF -- 0.0119 Glucose +0.0003 Glucose*BMI).

From the joint insulin level gamma fitted models (Table 1), the following outcomes can be reported. It is derived in the report that mean insulin level for $\mathrm{GW}$ is positively linked with glucose level $(\mathrm{P}=0.0055)$, concluding that insulin level increases as the glucose level rises. This is the natural functional role of insulin. It is negatively linked with age $(\mathrm{P}=0.0095)$, implying that insulin level decreases as at higher ages of $\mathrm{GW}$, which is observed in practice. Mean insulin level for GW is partially positively linked with Pregnancies*DPF $(\mathrm{P}=0.0864)$, implying that it increases as the joint effect of pregnancies and DPF increases. It shows that if pregnancies and DPF are higher, insulin level is also higher. Note that insulin level is independent of the marginal effects of pregnancies $(\mathrm{P}=0.1866)$ and $\mathrm{DPF}(\mathrm{P}=0.5321)$, while it is affected by their joint effect. Mean insulin level is positively linked with BP $(\mathrm{P}<0.0001)$ and TST $(\mathrm{P}<0.0001)$, while it is negatively linked with the joint interaction effect $\mathrm{BP} * \mathrm{TST}(\mathrm{P}<0.0001)$, implying that it increases as the BP and TST increase, but it can't increase proportionally as it is inversely linked with the joint effect $\mathrm{BP} * \mathrm{TST} \quad(\mathrm{P}<0.0001)$. In addition, it is inversely linked with the joint effect DPF*TST $(\mathrm{P}<0.0001)$, while DEF is independent of insulin level. Therefore, insulin level can't increase proportionally as TST, or BP increases. In addition, mean insulin level is negatively linked with BMI ( $\mathrm{P}=0.0001)$, implying that insulin level decreases as the BMI increases. Note that mean insulin level is positively partially linked with the interaction effect $\mathrm{BMI} * \mathrm{DPF}(\mathrm{P}=0.1312)$, while $\mathrm{DPF}$ is independent. 
Therefore, for GW with high BM along with high DPF, insulin level may increase a very little.

Variance of insulin level is positively linked with pregnancies $(\mathrm{P}=0.0184)$ and age $(\mathrm{P}=0.0027)$, while it is negatively linked with their joint interaction effect Pregnancies*Age $(\mathrm{P}=0.0418)$, implying that insulin level is not highly scattered at higher ages along with higher pregnancies. Again, variance of insulin level is partially negatively linked with BMI $(\mathrm{P}=0.0738)$, implying that insulin level is highly scattered for GW with lower BMI. Further, variance of insulin level is negatively linked with DPF ( $\mathrm{P}=0.0309)$, implying that insulin level is highly scattered for the GW with lower DPF values. Also, variance of insulin level is partially negatively linked with glucose level $(\mathrm{P}=0.1439)$, and it is partially positively linked with their joint interaction effect BMI*Glucose $(\mathrm{P}=0.1472)$, implying that insulin level variance has a very complicated relationship with the other factors. Partially significant factors are to be included in the models as confounders in Epidemiology.

The above stated results confirm many real situations as mentioned above. The article has many new results and joint interaction effects, which are rarely observed in any previous articles. Findings of the insulin level dispersion model are completely new to the insulin resistance study literature for GW. Earlier published articles have used multiple regression, analysis of variance, or logistic regression without dispersion model $[7,10,13]$. Most of the earlier articles' analysis outcomes have not been verified with the diagnostic checking, therefore, such results invite many doubts. The current outcomes can't be compared with the previous articles as they have not considered insulin level as a non-constant variance dependent variable. The present reported outcomes can be examined with the data set observed in the UCI Machine Learning Repository.

\section{Conclusions}

The current manuscript has derived the explanatory factors of insulin level for GW based on joint gamma fit along with the required model diagnostic checking. Final model is selected with the lowest AIC value, and based on the parameters having small standard errors (in Table 1), indicating that the estimates are stable. Therefore, the current research outcomes have a greater faith in presenting the real effects of insulin on GW. The similar data should present similar outcomes to the current results that are not shown herein as we have not any similar data in hand. The paper has derived a very complex insulin level relationship with the rest independent variables. The insulin level mean \& dispersion models have presented many uncommon outcomes, which are really new to the GW study literature. Medical researchers, GW and practitioners will be benefited from the report. It is concluded that for GW, insulin level increases with the increase of glucose level, BP, TST, and the interaction effects Pregnancies*DPF \& BMI*DPF, and it decreases with the increase age, BMI, and the interaction effects BP*TST \& DPF*TST. GW should be careful on her glucose level, BMI, BP \& TST regularly.

Conflict of interest: The authors confirm that this article content has no conflict of interest.

\section{References}

1. Das RN, Lee Y, Mukherjee S, Oh S. (2019). Relationship of body mass index with diabetes \& breast cancer biomarkers. J Diabetes and Management. 9(1): 163-168.

2. Mishra M, Ndisang JF. (2014). A critical and comprehensive insight on heme oxygenase and related products including carbon monoxide, bilirubin, biliverdin and ferritin in type-1and type-2 diabetes. Current Pharmaceutical Design. 20(9):13701391.

3. Petersen MC, Vatner DF, Shulman GI. (2017). Regulation of hepatic glucose metabolism in health and disease. Nature Reviews Endocrinology. 13(10): 572-587.

4. Marchetti P, Dotta F, Lauro D, Purrello F. (2008). An overview of pancreatic beta-cell defects in human type 2 diabetes: implications for treatment. Regul Pept. 146:4-11.

5. Kido Y, Nakae J, Accili D. (2001). Clinical review 125: The insulin receptor and its cellular targets. J Clin Endocrinol Metab.86: 972-979.

6. Savage DB, Petersen KF, Shulman GI. (2007). Disordered lipid metabolism and the pathogenesis of insulin resistance. Physiol Rev. 87:507-520.

7. Gray N, Picone G, Yashkin A. (2015). The relationship between BMI and onset of diabetes mellitus and its Complications. South. Med. J. 108(1): 29-36.

8. Valdez R, Mitchelj BD, Haffner SM, Hazuda HP, Morales PA, Monterrosa A, Stern MP. (1994). Predictors of weight change in a bi-ethnic population: the San Antonio Heart study. Int $\mathbf{J}$ Obes. 18: 85-91.

9. Odeleye OE, Courten M, Pettitt DJ, Ravussian E. (1997). Fasting hyperinsulinemia is a predictor of increased body weight and obesity in Pima Indian children. Diabetes. 46: 13411345.

10. Knowler WC, Pettitt DJ, Saad MF, Bennett PH. (1990). Diabetes mellitus in Pima Indians incidence risk factors and pathogenesis. Diabetes Metab. Rev. 6: 1-27.

11. Bennett PH, Burch TA, Miller M. (1971). Diabetes mellitus in American (Pima) Indians. Lancet. 2: 125-128.

12. Gray N, Picone G, Yashkin A. (2015). The relationship between BMI and onset of diabetes mellitus and its Complications. South. Med. J. 108(1), 29-36.

13. American Diabetes Association. (2006). Diagnosis and classification of diabetes mellitus. Diabetes. Care. 29: 43-81.

14. Das, RN. (2014). Determinants of Diabetes Mellitus in the Pima Indian Mothers and Indian Medical Students. The Open Diabetes Journal. 7, 5-13.

15. Myers RH, Montgomery DC, Vining GG. (2002). Generalized Linear Models with Applications in Engineering and the Sciences. New York: John Wiley \& Sons.

16. Firth D. (1988). Multiplicative errors: Log-normal or gamma? Journal of the Royal Statistical Society B. 50:266-268.

17. Das RN, Lee Y. (2009). Log-normal versus gamma models for analyzing data from quality-improvement experiments. Quality Engineering. 21(1): 79-87.

18. Lee Y, Nelder JA, PawitanY. (2017). Generalized Linear Models with Random Effects (Unified Analysis via Hlikelihood) (second edition). Chapman \& Hall, London.

19. Das M, An H, Chakraborty P, Aich AB. (2021). Relationship of full scale intelligence quotient (IQ) with verbal IQ, performance IQ \& physical characteristics. The International Journal of Latest Research in Science and Technology. 10(2): 19-23.

20. Das M. (2021). Induced Abortion Trends for Very Young Girls in New Zealand for the Period 2000--2019. Journal of Medicine: Study and Research. 4:019.

21. Hastie T, Tibshirani R, Friedman J. (2009). The Elements of Statistical Learning, Springer-Verlag.

22. Nelder JA. (1994). The statistics of linear models: back to basics. Statistics and Computing. 4:221-234. 